\title{
VOCALIZER F2: DESENVOLVIMENTO DE UM SCANNER DE MESA COM VOZ
}

\author{
Regina Heidrich \\ Universidade Feevale \\ rheidrich@feevale.br \\ Ana Paula Steigleder \\ Universidade Feevale \\ anapaulas@feevale.br \\ Juan Felipe Almada \\ Universidade Feevale \\ juanfa@feevale.br
}

\section{Resumo}

Este projeto tem como objetivo desenvolver um scanner de mesa que além de digitalizar o livro ou documento faça a leitura com voz. Autônomo, isso significa que funcionará sem estar ligado a nenhum equipamento. Para o desenvolvimento desta pesquisa escolhemos o estudo de caso pois o mesmo é uma investigação multifacetada, em profundidade, de um único fenômeno social. Consiste em uma pesquisa qualitativa, realizada por meio de pesquisa bibliográfica, documental e de estudo de caso, utilizando a metodologia de Cambridge Inclusive Design, viabilizado pela avaliação de usabilidade no desenvolvimento da interface de software e de produto. Pretende-se apresentar as fases de desenvolvimento de um produto realmente assistivo aos usuários com alguma limitação visual. Com os estudos apresentados nesse artigo, deseja-se posteriormente, desenvolver uma interface simples, intuitiva e que permita aos usuários cegos e com baixa visão, acessar com facilidade todos os recursos necessários à utilização do scanner.

Palavras-chave: Design Inclusivo, Ergonomia cognitiva, Interação, deficiência visual.

\begin{abstract}
This project aims to develop a flatbed scanner that, besides scanning a book or document, also offers a reading voice. Autonomic, meaning that it works without being connected to any equipment. For the development of this research we chose the case study because it is a multifaceted through research of a single social phenomenon. It consists of a qualitative research, carried out by means of literature, document and case study using the methodology of the Cambridge Inclusive Design, made possible by the usability evaluation in the development of software interface and product. We intend to present the stages of development of a product to users with
\end{abstract}


some visual impairment. With the studies presented in this article, we want to further develop a unique intuitive interface that allows blind users with low vision easy access to all the resources required to operate the scanner.

Keywords: Inclusive design, cognitive ergonomics, interaction, visual impairment.

\section{INTRODUÇÃO}

De acordo com dados do Censo 2010, mais de 35 milhões de brasileiros têm algum tipo de deficiência visual, sendo que mais de 6 milhões são totalmente cegos ou apresentam dificuldades severas (OLIVEIRA, 2012). Este projeto financiado pela FINEP, em parceria com uma empresa é composto por uma equipe interdisciplinar de 12 professores. São quatro doutores na área de Computação, Matemática, Engenharia Eletrônica e 8 doutorandos nas áreas de Design e Física. Além disso, o projeto conta com 5 bolsistas de iniciação científica. Tem como objetivo desenvolver um scanner de mesa que além de digitalizar o livro ou documento faça a leitura com voz. Autônomo, isso significa que funcionará sem estar ligado a nenhum equipamento.

Apesar de várias iniciativas do governo brasileiro no sentido de reduzir a lacuna entre pessoas com e sem deficiência, ainda faltam soluções para aumentar a autonomia dos deficientes visuais e facilitar seu acesso à informação, entretenimento, educação e trabalho. Ainda de acordo com o Censo 2010, quase metade dos brasileiros com 65 anos ou mais - 49,8\% - apresenta algum grau de deficiência visual (OLIVEIRA, 2012). A qualidade de vida da pessoa cega está diretamente ligada a possibilidade de ter condições de promover o desenvolvimento de suas potencialidades individuais. Assim o desenvolvimento de tecnologias assistivas voltadas a este público torna-se de fundamental importância para que se permita que o processo de inclusão escolar ocorra de fato.

Cook e Hussey definem a TA citando o conceito do ADA - American with Disabilities Act, como "uma ampla gama de equipamentos, serviços, estratégias e práticas concebidas e aplicadas para minorar os problemas funcionais encontrados pelos indivíduos com deficiências" (COOK \& HUSSEY, 1995).

O Comitê de Ajudas Técnicas - CAT ${ }^{1}$ do ministério da Ciência e Tecnologia do Brasil aprovou e reconheceu, em 14 de dezembro de 2007, este novo conceito:

Tecnologias Assistivas. É uma área do conhecimento, de característica interdisciplinar, que engloba produtos, recursos, metodologias, estratégias, práticas e serviços que objetivam promover a funcionalidade, relacionada a atividade e participação de pessoas com deficiência, incapacidades ou mobilidade reduzida, visando sua autonomia, independência, qualidade de vida e inclusão social.

O conceito de Tecnologia assistiva é considerado amplo, podendo ser peça chave na promoção dos Direitos Humanos, pois possibilita a pessoa com deficiência a oportunidade de alcançar autonomia e independência em diversos aspectos da sua vida (SECRETARIA DE DIREITOS HUMANOS DA PRESIDÊNCIA DA REPUBLICA, 2009).

\footnotetext{
${ }^{1}$ Disponível na internet por http em: <http://www.pessoacomdeficiencia.gov.br/app/sites/default/files/publicacoes/livro-tecnologiaassistiva.pdf>
} 
Deficiência é a ausência ou difusão de uma estrutura psíquica, fisiológica ou anatômica. A deficiência física causa uma desvantagem, resultante de um comprometimento ou de uma incapacidade, que limita ou impede o desempenho motor de determinada pessoa (Fernandes et al., 2007).

"Para as pessoas sem deficiência a tecnologia torna as coisas mais fáceis. Para as pessoas com deficiência, a tecnologia torna as coisas possíveis" (RADABAUGH, 1993).

\title{
2. DESENVOLVIMENTO
}

\subsection{Deficiência visual}

De acordo com a Organização Mundial de Saúde (WHO/OMS), "a cegueira é a incapacidade de ver". O cego vive em um mundo diferente do mundo do vidente, pois está desprovido de visão, luz e cor. É um mundo em que as informações recebidas pelos outros sentidos assumem maior importância (BLANCO, 2001). Assim, a cegueira é definida, num sentido clínico (BRUNO, 1997) ou legal (LIMA; NASSIF; FELIPPE, 2007), como a acuidade visual igual ou menor que 0,05 , com a melhor correção ótica (LIMA; NASSIF; FELIPPE, 2007; BRUNO, 1997) e "campo visual inferior a 20 graus" (BRUNO, 1997, p. 7). Já no sentido educacional, cegueira é a perda total de visão, chegando à nula projeção de luz, (BRUNO, 1997; BLANCO, 2001) "ou resíduo mínimo de visão que leva a pessoa a necessitar do sistema Braille [ou de computadores e suas funcionalidades] como meio de leitura e escrita." (LIMA; NASSIF; FELIPPE, 2007, p. 6).

Conforme Julião et al. (2013), a visão é responsável por fornecer informações para organização sensorial, compreender o mundo ao redor e dar significado aos objetos, conceitos e ideias. Portanto, a perda da capacidade visual implica a limitação das informações e conhecimentos que favorecem o desenvolvimento motor, perceptivo e emocional, causando adversidades de natureza individual e coletiva, que afetam a qualidade de vida, as restrições ocupacionais e a autoestima. Esses fatores podem, ainda, limitar o exercício da cidadania.

\begin{abstract}
Se para um grupo é uma questão de 'cabeça', psicológica ou criada por pessoas ditas 'normais', para outros é fato inegável que portar uma limitação física real e concreta expressa a dificuldade de realizar tarefas e funções 'comuns', por exemplo, as que dependem de locomoção. Para outros, ainda, representa a situação de transpor dificuldades e, por fim, tem aqueles que associam a eficiência especialmente ao mercado de trabalho (OLIVEIRA, 1999, p.107).
\end{abstract}

Desta maneira, Boa Ventura de Souza Santos (2003), aponta que o universalismo que queremos atualmente é aquele que tenciona a dignidade humana. Tendo este cerne, as diferenças surgem e devem ser respeitadas. "Temos direito de ser iguais quando a diferença nos inferioriza e direito de ser diferentes quando a igualdade nos descaracteriza. Daí a necessidade de uma igualdade que reconheça as diferenças e de uma diferença que não produza, alimente ou reproduza as desigualdades". (SANTOS, 2003, p. 56).

\subsection{Similares}

Realizou-se a análise de similares dos produtos existentes, a fim de conhecer suas características. A figura 1 (a) apresenta o scanner de mesa Scandock, que não necessita ser conectado a um computador para ser utilizado. A estrutura do produto 
apresenta uma aparência leve e de grande praticidade de manuseio. O usuário deve acoplar o seu celular smartphone na estrutura do scanner para captura das imagens. $O$ documento a ser digitalizado deve ser inserido na base de alumínio do produto, que apresenta uma inclinação de três graus. Na base de alumínio, existe uma película de silicone, oferecendo maior aderência ao documento a ser digitalizado e não permitindo dobras. As duas hastes posicionadas nas laterais, direita e esquerda distribuem uniformemente a luz sobre o documento a ser digitalizado, oito pequenas lâmpadas LED's estão inseridas nestas hastes a fim de distribuir a luz necessária para digitalização. O formato máximo para digitalização é de $22 \times 31 \mathrm{~cm}$. Os materiais utilizados para a produção deste produto foi o alumínio e polímero (SCANDOCK, 2016).

Figura 1: Imagens dos similares
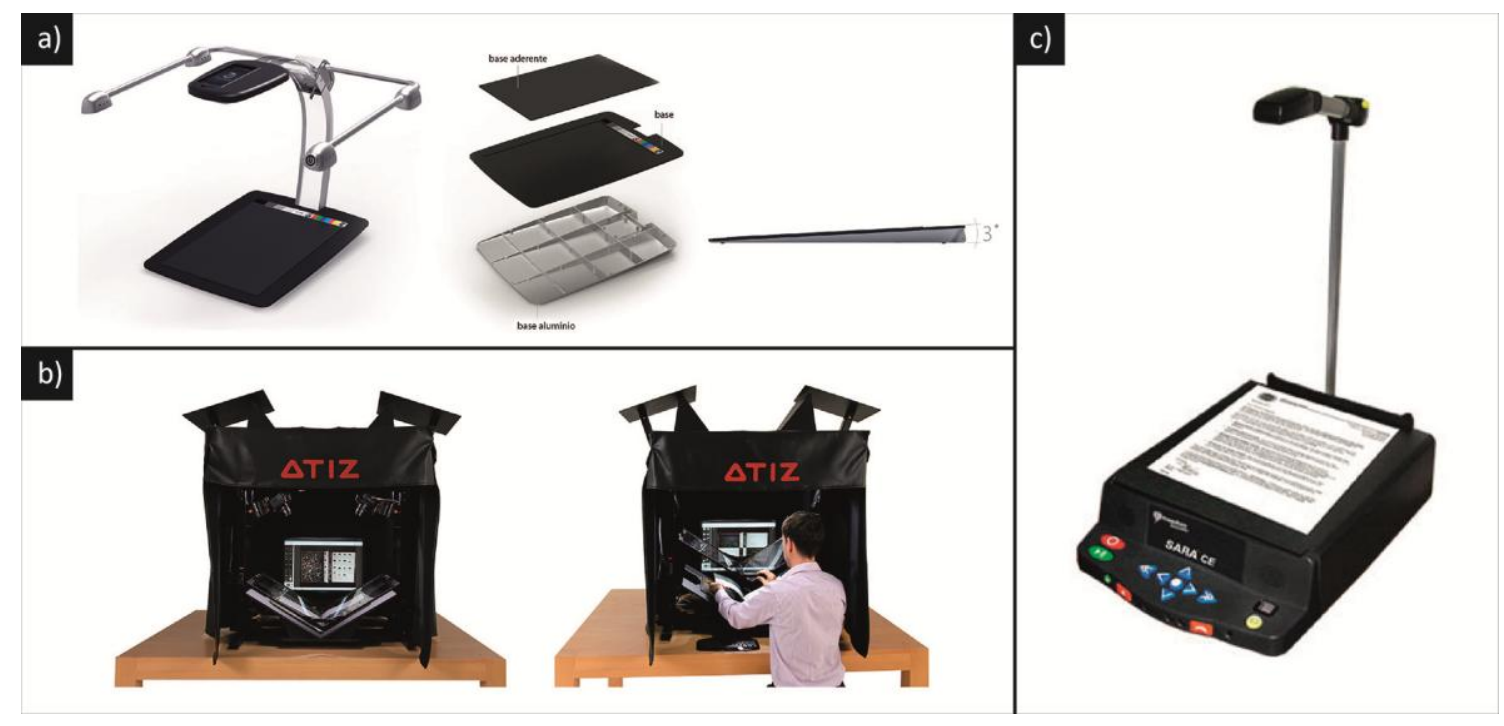

Fonte: Modificado de Scandock; Atiz; Freedom Scientific (2016)

Outro scanner que foi analisado foi o Book Drive Pro da Atiz, figura 01 (b). É um scanner de mesa que apresenta um tamanho maior, $130 \mathrm{~cm}$ de altura, $144 \mathrm{~cm}$ de largura e $138 \mathrm{~cm}$ de profundidade. Este scanner necessita ser conectado a um computador para ser utilizado. Um de seus diferenciais é a base de vidro em forma de um "V", que segundo os desenvolvedores, resolve o problema típico de curvatura dos livros no momento da digitalização chamado The Warping. Esse vidro preso ao livro aberto em ângulo de 120 graus, deixa suas páginas livres de curvaturas, facilitando assim seu escaneamento. Para digitalização são utilizadas duas câmeras, uma para a página da direita e outra para da esquerda. A captura das imagens é feita no mesmo momento para ambas as páginas. É possível capturar 800 páginas por hora. 0 formato máximo para digitalização é de 42 x 61,5cm (ATIZ, 2016).

O Sara CE, figura 1 (c) da Freedom Scientific, possui uma importante característica, de detectar automaticamente quando uma nova página é exposta a câmera. Para realizar a leitura em voz, este scanner necessita ser conectado a um computador. O teclado apresenta teclas grandes e táteis de fácil memorização. Esse scanner lê até 18 idiomas, além de apresentar outras características como aumento do tamanho do texto para quem possui baixa visão, mudar cor de fundo, adicionar mais espaço entre as letras, etc (FREEDOM SCIENTIFIC, 2016). 


\section{MATERIAIS E MÉTODOS}

Pesquisa de abordagem qualitativa. Para o desenvolvimento desta pesquisa escolhemos o estudo de caso pois o mesmo é uma investigação multifacetada, em profundidade, de um único fenômeno social. É conduzido em grande detalhe e, com frequência, se baseia no uso de várias fontes de dados. (FEAGIN; ORUM; SJOBERG, 1991).

Dentro da abordagem do Design usamos o Design Inclusivo e Design Centrado no Usuário. De acordo com ele British Standards Institute (2005) define o design inclusivo como "A concepção de produtos e / ou serviços de uso corrente, que são acessíveis e utilizáveis por, como muitas pessoas quanto possível, sem a necessidade de adaptação especial ou design especializado. O design inclusivo não sugere que é sempre possível projetar um produto para atender às necessidades de toda a população. Em vez disso, o design inclusivo orienta uma resposta de projeto adequada à diversidade da população. O design inclusivo não é design universal. Segundo Preiser e Ostroff (2001), o design para todos e o design universal têm o mesmo significado literal. Estas filosofias originadas, do design do ambiente construído e websites. No contexto do design de produto, tanto as abordagens do design para todos e do design universal fundamentalmente faz-se necessário aceitar que nem sempre é possível para, um produto, satisfazer as necessidades de toda a população. No entanto, estas abordagens sustentam que todos os produtos padronizados devem ser acessíveis a tantas pessoas quanto tecnicamente possível (PREISER e OSTROFF, 2001).

O Design Inclusivo definido por Clarkson et al. (2013), caracteriza-se por verificar e questionar a decisão de projeto de incluir ou excluir pessoas. $O$ design inclusivo ressalta a contribuição que a compreensão da diversidade de usuários proporcionará para o desenvolvimento do projeto. Abrange variação de capacidades, necessidades e aspirações e procura abranger o maior número possível de pessoas, conforme pode ser visualizado na figura 2.

Na fase pesquisar - OBSERVE USUÁRIOS. A observação do usuário é descobrir o que as pessoas realmente querem, o que eles realmente precisam, e o que eles realmente fazem. Pois os usuários, às vezes não têm consciência dos seus próprios hábitos e práticas, mudam o que dizem, dependendo do que eles acham que o entrevistador quer ouvir e não podem imaginar todas as possíveis alternativas para a situação atual.

Concentrando-se nas necessidades do usuário real é possível ajudar a evitar a sobrecarga da equipe de design do produto.. A observação do utilizador ajuda com outras atividades a explorar, bem como refinar as metas de produto. Ele deve ser complementado com outros métodos para descobrir as necessidades do usuário, tais como entrevistas, questionários, métodos de diário e grupos focais. Os dados antropométricos, ergonomia e capacidade também podem completar a observação do usuário para fornecer informações sobre a diversidade de usuários. Não geramos personas pois estamos trabalhando com usuários cegos.

A fase DESCREVER AS VIAGENS DOS USUÁRIOS consiste numa descrição passoa-passo da interação do usuário com um produto. Também descreve que o utilizador faz imediatamente antes e depois de utilizar o produto, uma vez que oferece algum contexto de utilização. Uma "viagem de usuário" ajuda a equipe de design para entender a experiência do usuário e ajuda a garantir que a lista de necessidades está completa. 
'Jornadas do usuário' devem, idealmente, ser construído por usuários observadores. As viagens podem ser descritas por escrever cada ação do usuário, e são reforçadas pela adição de fotografias. No nosso caso usamos fotografias e filmes.

Figura 2: Diagrama do Método de Design Inclusivo

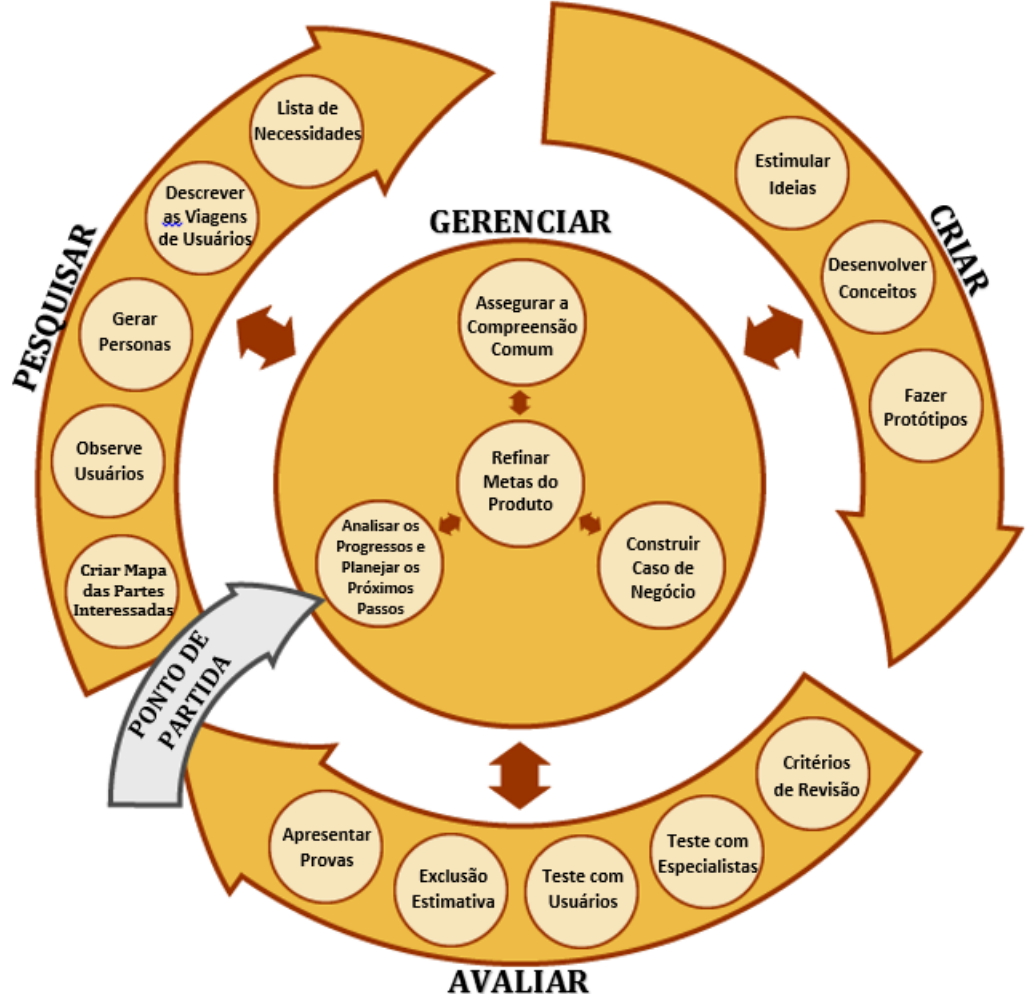

Fonte: Desenvolvido por Clarkson et al. (2013)

A fase LISTA DE NECESSIDADES é usada aqui para se referir a uma lista abrangente e categorizada. Cada necessidade pode ser capturada com uma declaração no formulário

Os resultados da lista de necessidades são: a) estabelecer uma ligação entre as exigências do projeto de design e as necessidades dos usuários; b) definição de prioridades das necessidades.

Usou-se inicialmente o Design Centrado no Usuário onde foram realizadas atividades de utilização de um scanner com voz, por uma pessoa cega. Os usuários interagem com um sistema através da sua interface. Os conceitos, as imagens e a terminologia apresentados na interface devem ser adequados às necessidades do usuário.

O design centrado no usuário parte do princípio que o usuário é o principal foco da realização de um produto, e prioriza as suas necessidades, desejos, expectativas, condicionamentos. Em cada etapa do projeto, designers e projetistas consultam pessoas representativas dos usuários finais, para conhecê-los em profundidade e aliar técnica e sensibilidade para resultados que criem empatia e identidade. (AVELLAR, DUARTE, 2016).

Realizou-se a experiência do usuário através do scanner Sara - PC (figura 3). Este scanner possui $25 \mathrm{~cm}$ de largura por $35 \mathrm{~cm}$ de profundidade e $3,5 \mathrm{~cm}$ de altura. Assim como os outros scanners, ele converte documentos impressos em voz. Utiliza 
reconhecimento óptico de caracteres OCR. Outra característica é que esse equipamento digitaliza também documentos em Braille, e esses posteriormente podem ser convertidos em voz. Assim como o Book Drive da Atiz, o Sara - PC necessita ser conectado a um computador. Seu mecanismo de funcionamento assemelha-se a um scanner comum, onde a tampa deve ser aberta e nesta abertura deverá ser inserido o documento a ser digitalização para posterior conversão em voz. $O$ tamanho da área de digitalização é de 21,5 x 29,7cm (FREEDOM SCIENTIFIC, 2016).

Figura 3: Experiência de usuário com Scanner Sara - PC
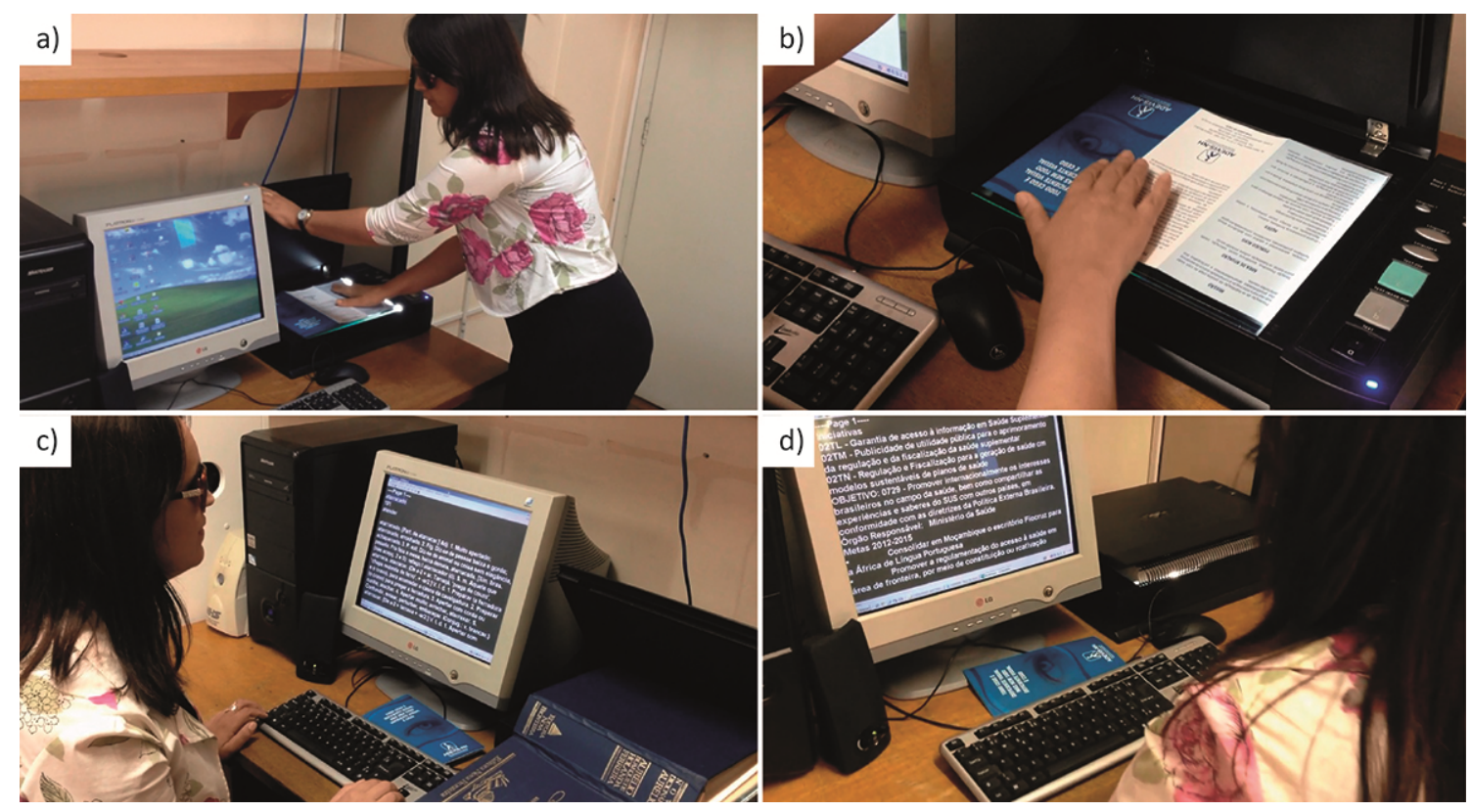

Fonte: Elaborado pelos autores, com base na pesquisa realizada (2016)

$\mathrm{Na}$ imagem (a) pode-se observar o usuário levantando a tampa e inserindo o documento no scanner. Antes deste processo, se faz necessário, tatear o dispositivo para localização da tampa e botões para o acionamento. Seguindo, na imagem (b), o usuário precisa ajustar o documento na bandeja do scanner. Em ambos procedimentos, verificou-se que a habilidade prévia do usuário auxiliou na execução da tarefa, mas um PCD despreparado apresentaria dificuldades para execução da tarefa. Nas imagens (c) e (d), pode-se observar o usuário utilizando o software que realiza a conversão do texto em voz e o escaneamento de diferentes materiais, enciclopédia e espiral, que oferecem maior dificuldade para o dispositivo realizar a captura da imagem.

\section{RESULTADOS}

Foi desenvolvido o primeiro protótipo de scanner de mesa, onde através dele os pesquisadores envolvidos neste projeto definiram parâmetros iniciais para o desenvolvimento do produto. Como pode ser observado na figura 4, o protótipo é dividido em duas partes, a base (a), e o topo (b). A base apresenta um formato de 80 $\mathrm{cm}$ de largura, por 53,5 cm de altura e $56 \mathrm{~cm}$ de profundidade. Tanto a base como 0 topo do protótipo foram pintados com tinta preta automotiva para não gerar refração de luz. Na base, existem duas portas, essas necessárias para a inserção dos quatro 
pontos de iluminação que serão manuseados dentro da base, esses fundamentais para gerar um cone de luz, para posterior captação das imagens. As lâmpadas foram instaladas em uma superfície de MDF de $12 \mathrm{~cm}$ de largura por $12 \mathrm{~cm}$ de profundidade e $3 \mathrm{~cm}$ de altura e logo um cano de PVC de $100 \mathrm{~mm}$ de diâmetro e 16,5 cm de altura colocado sobre a lâmpada figura 01 (b). No topo do cano de PVC foi acoplada uma lente da lupa Maped, esta que tem como objetivo potencializar o foco de luz.

\section{Figura 4: Protótipo}

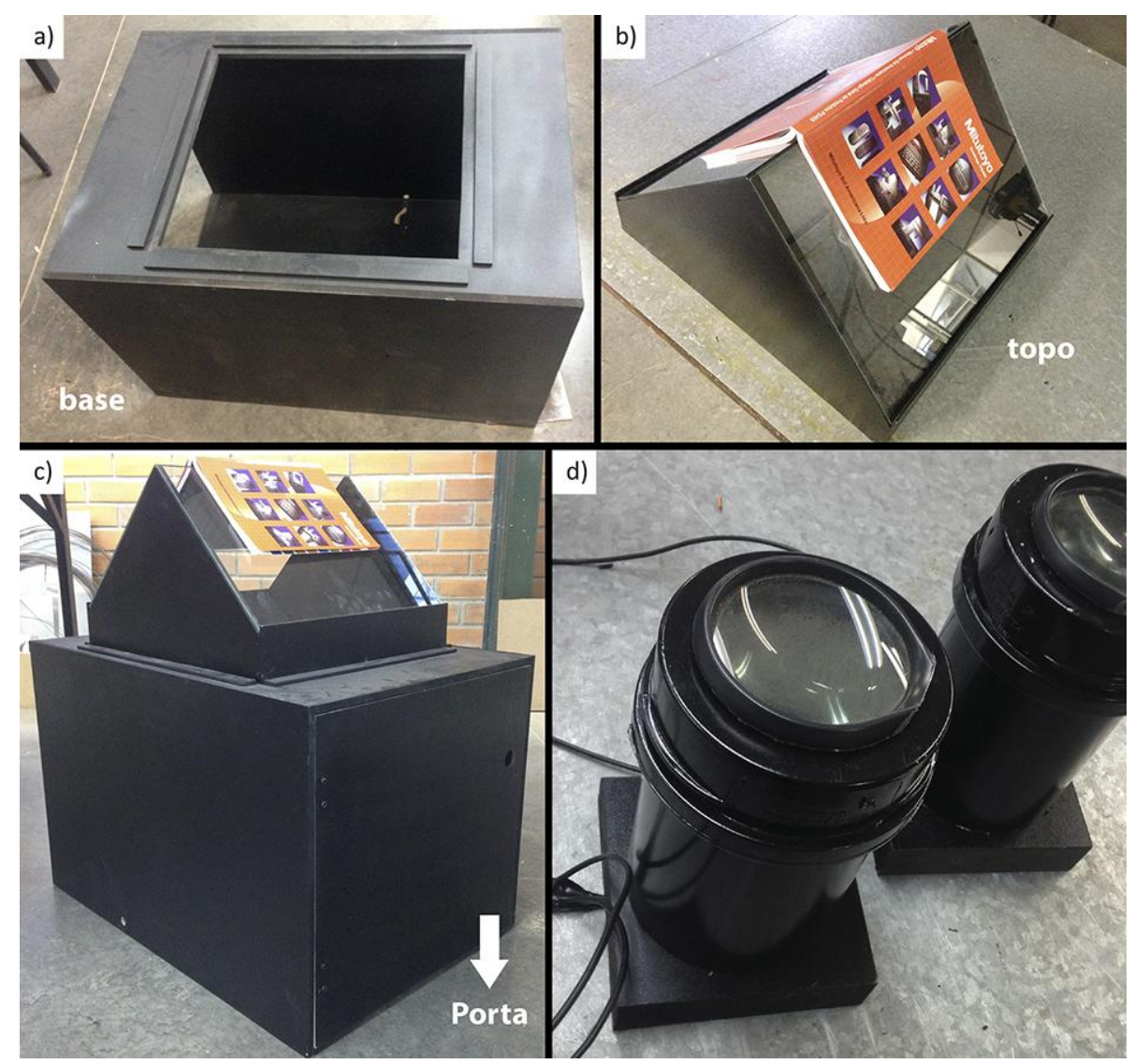

Fonte: Elaborado pelos autores, com base na pesquisa realizada (2016)

O topo do protótipo apresenta um formato de $50 \mathrm{~cm}$ de largura, $31 \mathrm{~cm}$ de altura e $45,5 \mathrm{~cm}$ de profundidade. O formato assemelha-se a uma pirâmide com ângulo de 45 graus. Duas lâminas de vidro de $42,7 \times 33 \mathrm{~cm}$ foram inseridas no topo da pirâmide, esta que terá a função de acomodar o livro, ou o material a ser digitalizado. Sugere-se esse formato, pois assim possivelmente eliminaremos problemas de leitura e escaneamento devido a curvatura de lombadas, wire-o (tipo de encadernação) e espirais utilizados para a prender as folhas destes materiais.

\section{CONCLUSÃO}

Os resultados obtidos até o momento caracterizam-se de um protótipo de um produto que será validado, e posteriormente adaptado. Através do trabalho realizado de uma equipe multidisciplinar, busca-se a simplicidade da solução, que oferecerá maior autonomia ao usuário. 
A análise de similares apresentada está sendo de grande relevância para o desenvolvimento da proposta gerada. Por intermédio deste estudo, verificou-se pontos a serem melhorados, para implementação da solução criada, para a partir disso, desenvolver uma proposta que possa superar os similares de mercado, oferecer autonomia para os usuários, consequentemente mais qualidade de vida.

\section{AGRADECIMENTOS}

Projeto financiado pela FINEP - Chamada Pública CTI/SECIS/FINEP/FNDCT Cooperação ICT - Empresa - Tecnologia Assistiva - 01/2013. Vocalizer F2: Desenvolvimento de um Scanner de mesa com voz.

\section{REFERÊNCIAS}

ATIZ. Book Drive Pro. Disponível em: http://pro.atiz.com. Acesso em: 13 maio 2016.

\section{AVELLAR e DUARTE. Design Centrado no Usuário.}

http://www.avellareduarte.com.br/layout/design-centrado-no-usuario/. Acesso em: 11 de maio 2016.

BLANCO, Maria Ángeles Núñez. Deficiencia Visual. III Congreso la atención a la diversidade en el sistema educativo. Universidad de Salamanca. Instituto Universitario de Integración en la Comunidad (INICO). 2001. Disponível em:

<http://campus.usal.es/ inico/actividades/actasuruguay2001/10.pdf>. Acesso em: 11 set. 2009.

BOGDAN, R.; BIKLEN, S. K. Qualitative research for education: an introduction for to theory and Methods. Boston: Allyn and Bacon, 1982.

BRASIL. Ministério de Ciência e Tecnologia. Ciência, Tecnologia e Inovação para o Desenvolvimento Nacional - Plano de Ação 2007-2010. Disponível em: . Acesso em: 7 set. 2009.

BRUNO, Marilda Moraes Garcia. Deficiência Visual: reflexão sobre a prática pedagógica. São Paulo, SP: Laramara, 1997.

. O Desenvolvimento Integral do Portador de Deficiência Visual. São

Paulo: Laramara, 1993.

CETINDAMAR, D.; PHAAL, R.; PROBERT, D. Understanding technology management as a dynamic capability: A framework for technology management activities.

Technovation, v. 29, n. 4, p. 237-246, 2009.

CLARKSON, P. J. et al. Inclusive design toolkit. 2007.

CLARKSON, P. John et al. Inclusive design: Design for the whole population. Springer Science \& Business Media, 2013.

COLEMAN, Roger et al. Design for inclusivity: a practical guide to accessible innovative and user-centred design. 2007.

COOK, A.M.; HUSSEY, S. M. Assistive Technologies: Principles and Practices. St. Louis: Mosby, 1995.

FEAGIN, J.; ORUM, A.; SJOBERG, G. A Case for the Case Study. Chapel Hill: The University of North Carolina Press, 1991. 
FERNANDES, A. C. et al. Medicina e Reabilitação: princípios e prática. São Paulo: Artes Médicas, 2007.

FREEDOM SCIENTIFIC. Sara CE. Disponível em:

http://www.freedomscientific.com/Content/Documents/ProductFlyers/SARA_CE_Flye r.pdf.Acesso em: 20 maio 2016.

IBGE. Censo Demográfico 2010: Características gerais da população, religião e pessoas com deficiência, maio 2012. Disponível em: http://censo2010.ibge.gov.br/resultados Acesso em: 08 junho 2016.

JULIÃO, Claudia Helena et al. A deficiência visual e o processo de construção da cidadania: um estudo no Instituto de Cegos do Brasil Central de Uberaba. Revista Família, Ciclos de Vida e Saúde no Contexto Social, v. 1, n. 1, 2013.

LIMA, Eliana Cunha; NASSIF, Maria Christina Martins; FELIPPE, Maria Cristina Godoy Cruz. Convivendo com a Baixa Visão: da criança à pessoa idosa. São Paulo: Fundação Dorina Nowill para Cegos, 2007.

OLIVEIRA, M.L.B. Cartilha do Censo 2010 - Pessoas com Deficiência. Secretaria de Direitos Humanos da Presidência da República (SDH/PR) / Secretaria Nacional de Promoção dos Direitos da Pessoa com Deficiência (SNPD) / Coordenação-Geral do Sistema de Informações sobre a Pessoa com Deficiência; Brasília: SDH-PR/SNPD, 2012.

PATTON, M. Q. How to use qualitative Methods in Evaluation. London: Sage, 1987.

OLIVEIRA, Cristiane Cardoso de. 0 trabalhador portador de deficiência física e sua inclusão no mundo do trabalho. 1999. Dissertação (Mestrado em Serviço Social) Faculdade de Serviço Social, Pontifícia Universidade Católica, Porto Alegre.

PREISER and OSTROFF. Inclusive Design Tool Kit. Disponível em: http://www.inclusivedesigntoolkit.com/betterdesign2/whatis/whatis.html Acesso em: 08 de junho de 2016.

RADABAUGH, M. P. Study on the Financing of Assistive Technology Devices of Services for Individuals with Disabilities -A report to the president and the congress of the United State, National Council on Disability, Março 1993. Disponível em Acesso em 04 dez. 2007.

SANTOS, Boaventura de Sousa. Reconhecer para libertar: os caminhos do cosmopolitanismo multicultural. Introdução: para ampliar o cânone do reconhecimento, da diferença e da igualdade. Rio de Janeiro: Civilização Brasileira, 2003: 56.

SCANDOCK. Scandock. Disponível em: http://www.scandock.com Acesso em: 12 maio 2016.

UNIVERSITY OF WASHINGTON. What is assistive technology? Disponível em: < USABILITY.GOV. Acesso em: 22 jul. 2008.

YIN, R. K. Case Study Research: Design and Methods. 2. ed. Thousand Oaks: Sage, 1994. 